\title{
IL-6 Plays Crucial Roles in Sporadic Colorectal Cancer through the Cytokine Networks including CXCL7
}

\author{
Takashi Uchiyama $^{1}$, Hirokazu Takahashi ${ }^{1}$, Hiroki Endo ${ }^{1}$, Eiji Sakai $^{1}$, Kunihiro Hosono ${ }^{1}$, \\ Yoji Nagashima $^{2}$, Atsushi Nakajima ${ }^{1^{*}}$ \\ ${ }^{1}$ Division of Gastroenterology, Yokohama City University School of Medicine, Yokohama, Japan; ${ }^{2}$ Department of Molecular Pa- \\ thology, Yokohama City University School of Medicine, Yokohama, Japan. \\ Email: "nakajima-tky@umin.ac.jp
}

Received September $9^{\text {th }}, 2012$; revised October $11^{\text {th }}, 2012$; accepted October $20^{\text {th }}, 2012$

\begin{abstract}
IL-6 is a multifunctional cytokine and involved in variety of carcinogenesis. However, the association between IL-6 and sporadic colorectal cancer has not been fully explained. Here, we investigated the role of IL-6 signaling and the cytokine network in sporadic colorectal cancer. We investigated the serum IL-6 levels in patients with sporadic colorectal adenoma, cancer patients, and normal controls. In addition, the expressions of IL-6, gp130, and the IL-6 receptor subunit were investigated in biopsy specimens collected from these subjects. Furthermore, the expressions of CXCL7 and CXCR2, a chemokine and its receptor involved in IL-6 production, were also investigated. We observed an elevated level of serum IL-6 in colorectal cancer patients and an increased expression of IL-6 in colorectal cancer tissues, compared with the levels in a control group and in patients with adenoma. The phosphorylation of gp130 was also increased in the colorectal cancer tissues, compared with that in control and adenoma tissues. The expressions of CXCL7 and CXCR2 in the colorectal cancer tissues were also higher than those in control and adenoma tissues. IL-6 signaling is involved in sporadic colorectal cancer. In addition, the increased expressions of CXCL7 and CXCR2 might, in turn, increase the expression of IL-6 in colorectal cancer. Further studies are required to elucidate the function of the IL-6 signaling and the cytokine network in sporadic colorectal cancer.
\end{abstract}

Keywords: IL-6; Colorectal Cancer; gp130; CXCL7

\section{Introduction}

Colorectal cancer is a major cause of mortality and morbidity worldwide [1], although the mechanism of colorectal carcinogenesis remains unclear. Many studies have reported an association between interleukin-6 (IL-6) and colitis-associated cancer [2-4]. On the other hand, the association between IL-6 signaling and the sporadic colorectal adenoma-carcinoma sequence has not yet been clarified. IL-6 is a multifunctional cytokine important for immune response, cell survival, apoptosis and proliferation. IL-6 binds to soluble or membrane-bound IL-6 receptor (IL-6R) polypeptides that signal by interacting with the membrane-associated gp130 subunit, the engagement of which triggers the activation of Janus kinases (JAK)/STAT3 signaling [5]. Previous study showed that the interaction between mesenchymal stem cells and cancer stem cells is mediated by a positive feedback cytokine loop in which IL6 and CXCL7 play pivotal roles in vivo [6]. Chemokines are a family of small molecular weight proteins. CXCL7 is a member of the CXC sub-

\footnotetext{
${ }^{*}$ Corresponding author.
}

family of chemokines which can be further subdivided on the basis of the presence of the tripeptide motif glutamate-leucinearginine. The expression of CXCL7 and its receptor CXCR2 has been shown to be increased in breast cells [7]. However, the cytokine network in sporadic colorectal adenoma-carcinoma sequence has not been previously reported. Therefore, in the present study, we investigated IL-6 signaling and the cytokine network in the colorectal cancer patients.

\section{Materials and Methods}

\subsection{Study Population}

Thirty patients who were diagnosed as having colorectal adenoma, 30 patients with cancer, and 20 control subjects who were confirmed not to have any colorectal polyps when examined using a colonoscopy were recruited for this study between 2008 and 2009 at Yokohama City University Hospital. The exclusion criteria were subjects with familial adenomatous polyposis, inflammatory bowel disease, radiation colitis, or any malignant disease, as well as subjects with a previous his- 
tory of colectomy, gastrectomy or colorectal polypectomy. Written informed consent was obtained from all the subjects prior to their participation in the study. The study protocol was approved by the Yokohama City University Hospital Ethics Committee.

\subsection{Collection and Analysis of Blood Samples for the Determination of IL-6 Levels}

Blood samples were obtained in the morning on the day of the colonoscopy after the subjects had fasted overnight. The serum IL-6 levels were measured using an enzymelinked immunosorbent assay for human IL-6 (SRL Co., Tokyo, Japan).

\subsection{Immunohistochemical Analyses}

The expressions of IL-6 and gp130 were investigated in normal colorectal, adenoma, and cancer tissues. Samples of normal colorectal, adenoma and cancer tissues were obtained from the study subjects and isolated. Formalin-fixed and paraffin-embedded samples were later deparaffinized and rehydrated. The sections were incubated with primary antibodies for IL-6 (1:50; Leica Biosystems, Mount Waverley, Victoria, Australia) and gp130 (1:100; Santa Cruz Biotechnology, Santa Cruz, California, USA) using an LSAB2 kit (DakoCytomation, Glostrup, Denmark). The sections were then incubated with biotinylated immunoglobulin as the secondary antibody and treated with peroxidase-conjugated streptavidin. The antibody complex was visualized with 3,3'-diaminobenzidine, tetrahydrochloride (Dojindo Laboratories, Kumamoto, Japan).

\subsection{Western-Blot Analysis}

Samples obtained from normal colorectal, adenoma and cancer tissues were isolated. The extracted protein was separated using sodium dodecylsulfate polyacrylamide gel electrophoresis (SDS-PAGE), and the separated proteins were transferred to a polyvinylidene difluoride (PVDF) membrane (Amersham, London, UK). The membranes were probed with primary antibodies specific for phospho-gp130 (p-gp130) and gp130 (Santa Cruz Biotechnology). Horseradish peroxidase-conjugated secondary antibodies and the ECL detection kit (Amersham) were used to detect specific proteins.

\subsection{Real-Time RT-PCR}

Samples obtained from the normal colorectal, adenoma and cancer tissues were isolated. Total RNA from the colorectal cancer and normal colorectal tissues were extracted using the RNeasy Mini Kit (Qiagen, Hilden, Germany). For the real-time reverse-transcriptase polymerase chain reaction, total RNA was reverse-transcribed into cDNA and amplified using a real-time quantitative polymerase chain reaction using the Step One Plus Real Time PCR System (Applied Biosystems, Foster City California, USA). Probes and primer pairs specific for IL-6, CXCL7, CXCR2 and 18S were purchased from Applied Biosystems. The concentrations of the target genes were determined using the competitive computed tomography method, and the values were normalized to the internal control.

\subsection{Statistical Analysis}

Statistical analyses were performed using the KruskalWallis test and the Fisher PLSD test. All analyses were performed using StatView software (SAS Institute, Cary, NC, USA). A value of $p<0.05$ was regarded as denoting statistical significance.

\section{Results}

\subsection{Serum IL-6 Levels in Study Subjects and Expression of IL-6 in Normal Colorectal, Adenoma, and Cancer Tissues}

The clinical characteristics of the colorectal cancer patients and the control subjects are shown in Table 1. No significant differences in age, BMI, or serum CRP levels were observed among these groups. The serum IL-6 level was significantly higher among the colorectal cancer patients than among the normal colorectal subjects or the adenoma patients $(p<0.05)$, although no significant difference was observed between the normal colorectal subjects and the adenoma patients (Figure 1(a)). To examine the expression of IL-6 in normal colorectal, adenoma and cancer tissues, immunohistochemical staining and gene expression analyses were performed. The expression of IL-6 was observed in normal colorectal, adenoma, and cancer tissues (Figures 1(c)-(e)). The mRNA expression of IL-6 was significantly higher in the colorectal cancer tissues than in the normal colorectal or adenoma tissues $(p<0.01)$. No significant difference was observed between the normal colorectal and adenoma tissues (Figure 1(b)).

\subsection{Expression and Phosphorylation of gp130 in Colorectal Tissues}

IL-6 acts via a receptor complex containing at least one subunit of the signal-transducing protein gp130 [8]. We also investigated the expression of gp130 in normal colorectal, adenoma and cancer tissues using immunohistochemical staining and examined the phosphorylation of gp130 using a western blot analysis. The expression of gp130 was observed in normal colorectal, adenoma, and cancer cells (Figures 2(a)-(c)). The phosphorylation of gp130 was significantly higher in the colorectal cancer 
Table 1. Characteristics of the study patients.

\begin{tabular}{ccccc}
\hline & Normal & Adenoma & Cancer & $p$ value \\
\hline $\mathrm{N}$ & 20 & 30 & 30 & \\
\hline Age (year) & $66.8 \pm 11.4$ & $65.2 \pm 9.6$ & $68.6 \pm 12.0$ & 0.30 \\
Sex m/f & $13 / 7$ & $27 / 15$ & $15 / 6$ & 0.34 \\
BMI $\left(\mathrm{kg} / \mathrm{m}^{2}\right)$ & $21.9 \pm 3.1$ & $13.4 \pm 2.8$ & $22.9 \pm 4.2$ & 0.21 \\
CRP $(\mathrm{mg} / \mathrm{dl})$ & $0.1 \pm 0.2$ & $0.3 \pm 0.9$ & $1.2 \pm 2.4$ & 0.12 \\
\hline
\end{tabular}

Data are shown as mean \pm standard deviation (SD).

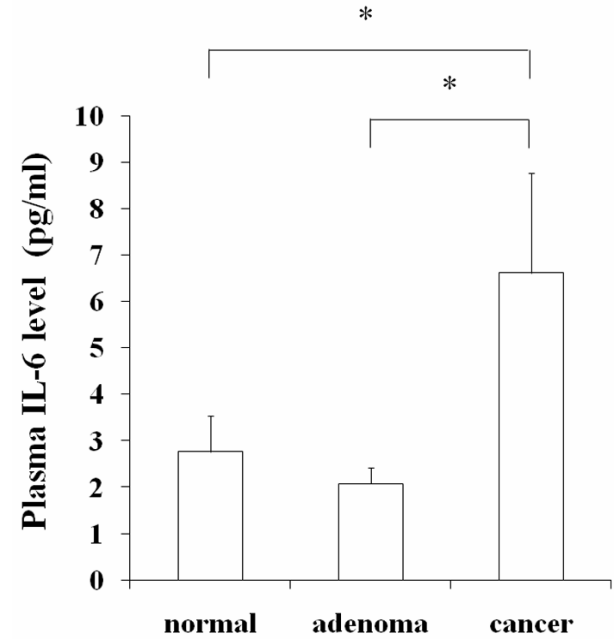

(a)

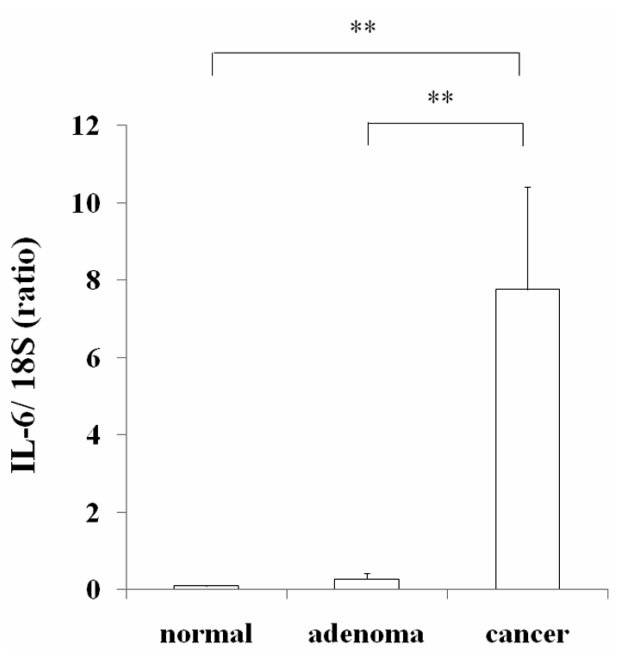

(b)

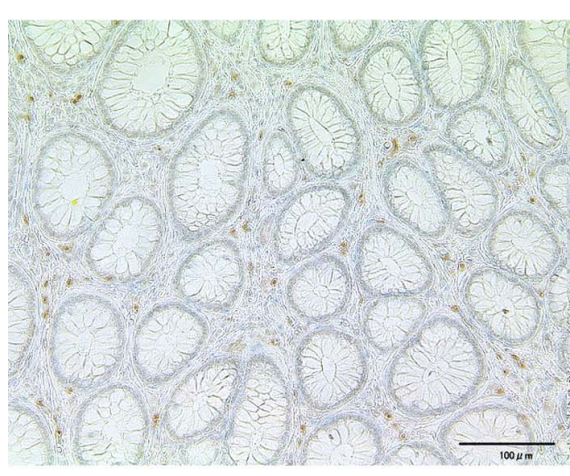

(c)

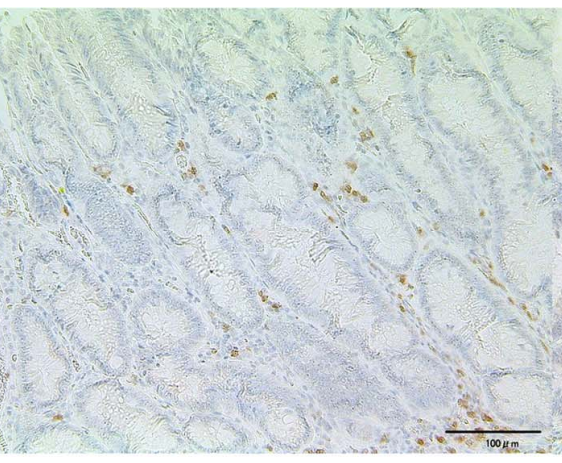

(d)

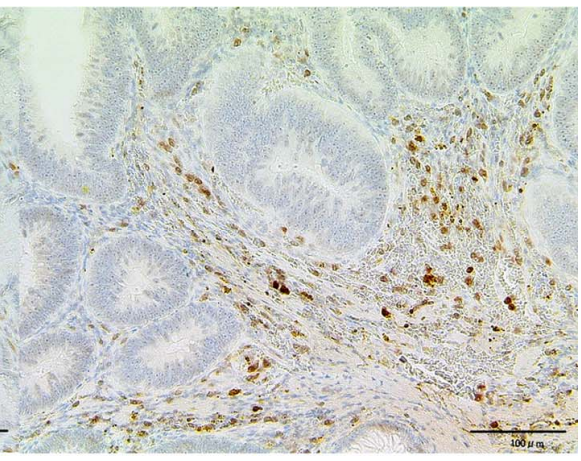

(e)

Figure 1. (a) Serum IL-6 levels in normal control, colorecdtal adenoma and cancer patients were expressed. Each column represents the mean \pm SEM. ${ }^{*} p<0.05,{ }^{* *} p<0.01$; (b) The relative mRNA expressions of IL-6 in normal colorectal, adenoma and cancer tissues were expressed as the ratio relative to the expression of $18 \mathrm{~S}$. Each column represents the mean \pm SEM. ${ }^{*} p$ $<0.05,{ }^{* * *} p<0.01$; ((c)-(e)) Immunohistochemical staining for IL-6. Scale bar: $100 \mu \mathrm{m}$; (c) Normal colorectal tissue; (d) Colorectal adenoma tissue; (e) Colorectal cancer tissue.

tissues than in the normal and adenoma tissues (Figure 2(d)). No significant difference was observed between the normal colorectal and adenoma tissues.

\subsection{Expressions of CXCL7 and CXCR2 in Colorectal Tissues}

CXCL7 is a member of the CXC subfamily of chemokines, and its receptor is CXCR2. The mRNA expression levels of CXCL7 and CXCR2 were significantly higher in the colorectal cancer tissues than in the normal colorectal or colorectal adenoma tissues (Figure 3). No sig nificant difference was observed between the normal colorectal and adenoma tissues.

\section{Discussion}

Previous studies have reported an association between 


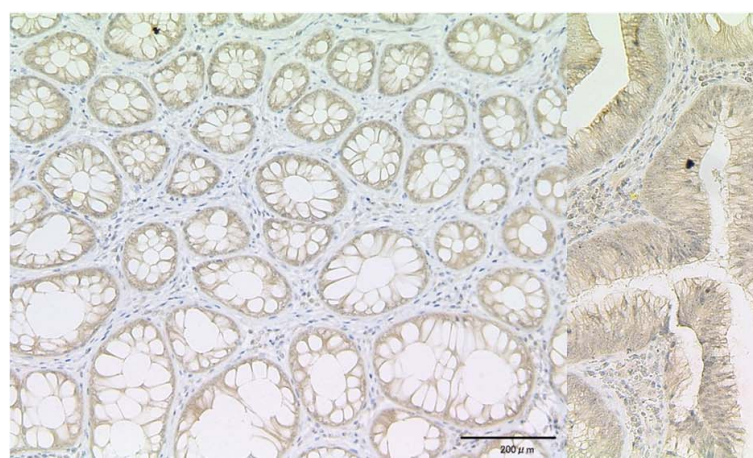

(a)

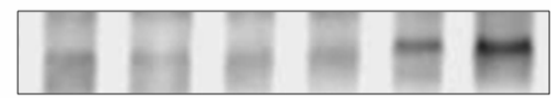

p-gp130

gp130

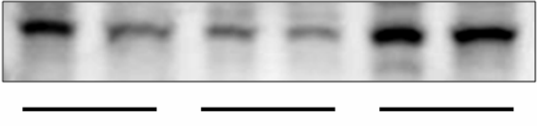

normal

adenoma

cancer

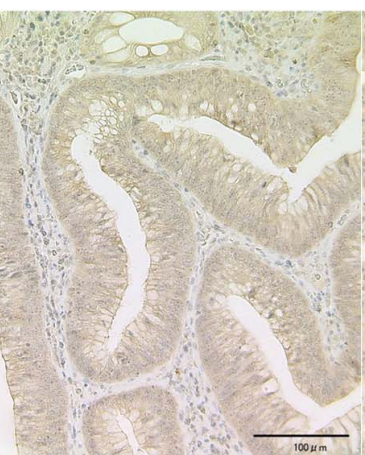

(b) (c)

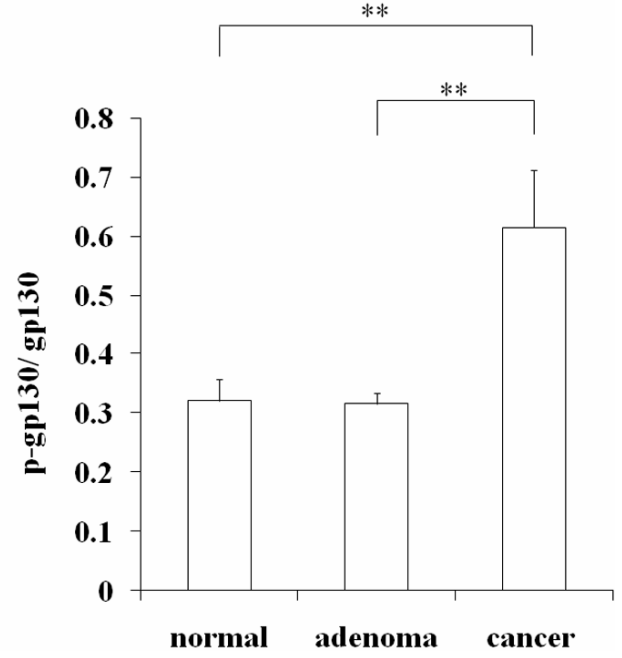

(d)

Figure 2. ((a)-(c)) Immunohistochemical staining for gp130. Scale bar: $100 \mu \mathrm{m}$. (a) Normal colorectal tissues; (b) Colorectal adenoma tissues; (c) Colorectal cancer tissues; (d) WESTERN-blot analysis for gp130 and phosphorylated gp130. Left panels: Representative Western blots for phosphorylated and total levels of gp130. Lanes 1, 2: normal colorectal tissue; lanes 3, 4: adenoma tissue; lanes 5, 6: cancer tissue. Right panels: The ratios of the level of the phosphorylated gp130 to the total gp130 level. Each column represents the mean \pm SEM. ${ }^{*} p<0.05,{ }^{* * *} p<0.01$.

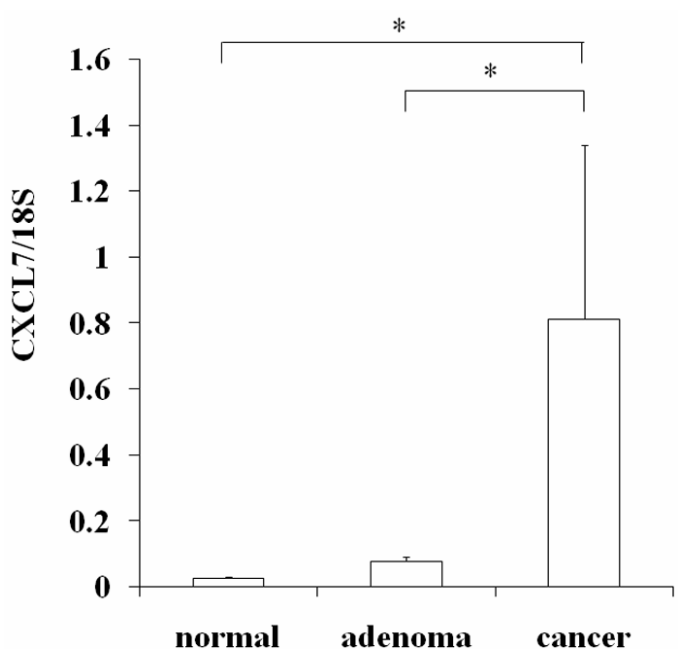

(a)

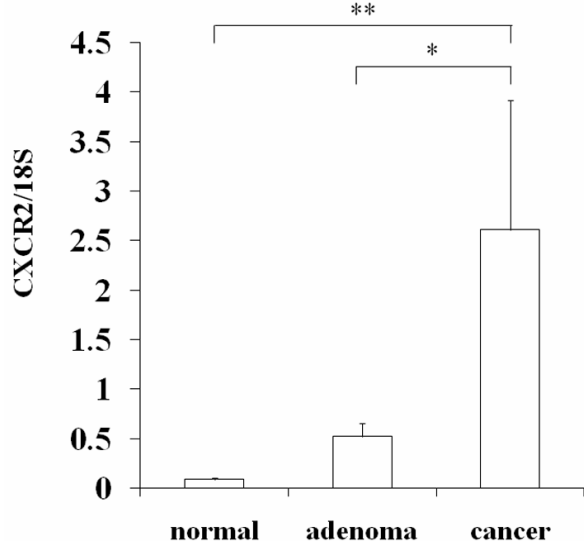

(b)

Figure 3. The relative mRNA expressions of (a) CXCL7 and (b) CXCR2 in normal colorectal, colorectal adenoma and cancer tissues are expressed as the ratios relative to the expression level of $18 \mathrm{~S}$. Each column represents the mean $\pm \mathrm{SEM}$. ${ }^{*} p<0.05$, ${ }^{* * *} p<0.01$. 
IL-6 and CAC [2-4], although the relation between IL-6 and the sporadic colorectal adenoma-carcinoma sequence has not been fully explained. In the present study, we investigated IL-6 signaling in the sporadic colorectal adenoma-carcinoma sequence. We observed a significant increase in the serum IL-6 level in cancer patients with colorectal cancer, compared with normal subjects and patients with adenoma. In addition, the level of IL-6 expression was higher in colorectal cancer tissues than in normal or adenoma tissues. Thus, we speculated that IL-6 may play crucial roles in not only CAC, but also sporadic colorectal cancer. No significant difference was observed in the serum CRP levels among the groups. This result indicated that the increase in IL-6 expression was not mediated by systematic inflammation.

We investigated the phosphorylation level of gp130, which is mediated by IL-6 signaling in colorectal tissues. We observed significantly higher gp130 phosphorylation levels in colorectal cancer tissues than in normal or adenoma tissues. To the best of our knowledge, this is the first study that demonstrates the expression of the gp130 phosphorylation in human colon cancer tissue. IL-6 mediates JAK/STAT signaling is involved in cell proliferation $[5,9,10]$. Previous studies have revealed that the phosphorylation of STAT3 is higher in colorectal cancer tissues than in normal or adenoma tissues [11-14]. Combined with these previous studies, our present results suggested that IL-6 mediates JAK/STAT signaling thorough the phosphorylation of gp130 in sporadic colorectal cancer, but not in adenoma or normal colorectal tissues.

In addition, we observed an increase in CXCL7 and CXCR2 expression in colorectal cancer tissues. Mesenchymal stem cells derived CXCL7, in turn, interacts with cancer cells through CXCR2, inducing the synthesis of a number of cytokines, including IL-6 [15]. Previous studies have reported elevated expression levels of CXCL7 and CXCR2 [7,15], and the formation of a positive feedback loop between increases in IL-6 and CXCL7 has been suggested in breast cancer [6]. However, the expression levels of CXCL7 and CXCR2 have not been previously studied in colorectal cancer. Although we could not show direct evidence of this signaling in human colorectal adenomas, our results were consistent with previous studies in breast cancer; thus, we speculated that IL-6 and CXCL7 may also form a positive feedback loop in sporadic colon cancer. This mechanism would be a novel finding in colon cancer. We propose that this cytokine network may be a novel therapeutic target for the prevention of colorectal cancer.

In conclusion, IL-6 may play a crucial role in the sporadic colorectal cancer. The increased expressions of CXCL7 and CXCR2 might, in turn, increase the expression of IL-6 in colorectal cancer. Further investigations are required to clarify the exact roles of IL-6 signaling in sporadic colorectal cancer.

\section{Acknowledgements}

We thank Machiko Hiraga for her technical assistance. This work was supported in part by a Grant-in-Aid for research on the Third-Term Comprehensive Control Research for Cancer from the Ministry of Health, Labour and Welfare, Japan, to A. N., a grant from the National Institute of Biomedical Innovation (NBIO) to A. N., a grant from the Ministry of Education, Culture, Sports, Science and Technology, Japan (KIBAN-B) to A. N., and the grant program, "Collaborative Development of Innovative Seed" from the Japan Science and Technology Agency (JST).

\section{REFERENCES}

[1] A. Jemal, R. Siegel, J. Xu and E. Ward, "Cancer Statistics, 2010,” CA: A Cancer Journal for Clinicians, Vol. 60, No. 5, 2010, pp. 277-300. doi.org/10.3322/caac.20073

[2] E. Langholz, P. Munkholm, M. Davidsen and V. Binder, "Colorectal Cancer Risk and Mortality in Patients with Ulcerative Colitis,” Gastroenterology, Vol. 103, No. 5, 1992, pp. 1444-1451.

[3] W. Holtkamp, T. Stollberg and H. E. Reis, "Serum Interleukin-6 Is Related to Disease Activity but Not Disease Specificity in Inflammatory Bowel Disease," Journal of Clinical Gastroenterology, Vol. 20, No. 2, 1995, pp. 123-126. doi:10.1097/00004836-199503000-00010

[4] C. Niederau, F. Backmerhoff, B. Schumacher and C. Niederau, "Inflammatory Mediators and Acute Phase Proteins in Patients with Crohn's Disease and Ulcerative Colitis,” Hepatogastroenterology, Vol. 44, No. 13, 1997, pp. 90-107.

[5] T. Kishimoto, "Interleukin-6: From Basic Science to Medicine-40 Years in Immunology," Annual Review of Immunology, Vol. 23, 2005, pp. 1-21. doi:10.1146/annurev.immunol.23.021704.115806

[6] S. Liu, C. Ginestier and S.J. Ou, "Breast Cancer Stem Cells Are Regulated by Mesenchymal Stem Cells through Cytokine Networks,” Cancer Reserch, Vol. 71, No. 6, 2011, pp. 614-624. doi:10.1158/0008-5472.CAN-10-0538

[7] Z. Tang, M. Yu, F. Miller, R. S. Berk, G. Tromp and M. A. Kosir, "Increased Invasion through Basement Membrane by CXCL7-Transfected Breast Cells,” The American Journal of Surgery, Vol. 196, No. 5, 2008, pp. 690696. doi:10.1016/j.amjsurg.2008.08.001

[8] T. Taga and T. Kishimoto, "Gp130 and the Interleukin-6 Family of Cytokines”, Annual Review of Immunology, Vol. 15, 1997, pp. 797-815. doi:10.1146/annurev.immunol.15.1.797

[9] Z. Zhong, Z. Wen and J. E. Darnell Jr., "Stat3: A STAT Family Member Activated by Tyrosine Phosphorylation in Response to Epidermal Growth Factor and Inter- 
leukin-6,” Science, Vol. 264, No. 5155, 1994, pp. 95-98. doi:10.1126/science.8140422

[10] T. Kordula, M. Bugno, J. Goldstein, and J. Travis, “Activation of Signal Transducer and Activator of Transcription-3 (Stat3) Expression by Interferon-Gamma and Interleukin-6 in Hepatoma Cells," Biochemical and Biophysical Research Communications, Vol. 216, No. 3, 1995, pp. 999-1005. doi.org/10.1006/bbrc.1995.2719

[11] T. Kusaba, T. Nakayama, K. Yamazumi, Y. Yakata, A. Yosizaki, T. Nagayasu and I. Sekine, "Expression of p-Stat3 in Human Colorectal Adenocarcinoma and Adenoma; Correlation with Clinicopathological Factors," Journal of Clinical Pathology, Vol. 58, No. 8, 2005, pp. 833-838. doi:10.1136/jcp.2004.023416

[12] J. K. Park, R. Hong, K. J. Kim, T. B. Lee and S. C. Lim, "Significance of p-Stat3 Expression in Human Colorectal Adenocarcinoma," Oncology Reports, Vol. 20, No. 3, 2008, pp. 597-604. doi:10.3892/or00000047

[13] X. T. Ma, S. Wang, Y. J. Ye, R. Y. Du, Z. R. Cui and M.
Somsouk, "Constitutive Activation of Stat3 Signaling Pathway in Human Colorectal Carcinoma,” World Journal of Gastroenterology, Vol. 10, No. 11, 2004, pp. 15691573.

[14] F.M. Corvinus, C. Orth, R. Moriggl, S. A. Tsareva, S. Wagner, E. B. Pfitzner, D. Baus, R. Kaufmann, L. A. Huber, K. Zatloukal, H. Beug, P. Ohlschläger, A. Schütz, K. J. Halbhuber and K. Friedrich, "Persistent STAT3 Activation in Colon Cancer Is Associated with Enhanced Cell Proliferation and Tumor Growth,” Neoplasia, Vol. 7, No. 6, 2005, PP. 545-555. doi:10.1593/neo.04571

[15] G. Kalwitz, M. Endres, K. Neumann, K. Skriner, J. Ringe, O. Sezer, M. Sittinger, T. Häupl and C. Kaps, "Gene Expression Profile of Adult Human Bone Marrow-Derived Mesenchymal Stem Cells Stimulated by the Chemokine CXCL7,” The International Journal of Biochemistry \& Cell Biology, Vol. 41, No. 3, 2009, 649-658. doi.org/10.1016/j.biocel.2008.07.011 